\title{
Transmission - Sonifying, Visualising and Analysing Neural Activity through Telepresence
}

\author{
Oliver Gingrich \\ Bournemouth University \\ London W9 3EN, United Kingdom \\ oliver@musion.co.uk
}

\author{
Evgenia Emets \\ London \\ United Kingdom \\ emetsjane@gmail.com
}

\author{
Alain Renaud \\ Bournemouth University \\ Geneva, Switzerland \\ arenaud@bournemouth.ac.uk
}

\begin{abstract}
Transmission is a telematic performance which allows for real time sonification and visualisation of brain waves and looks at the effect of interaction on the human consciousness. Users are equipped with an Emotiv EEG which enables them to perceive sonification and visualisation of their brain's processing in real time. This visualisation is modified through another user's movement in a remote location - the user's consciousness becomes a new musical and visual interface played by a remote person. Transmission is both a research project on telepresence and a participatory audience tool.
\end{abstract}

Telepresence. Neuro-activity. Neurofeedback. Brain wave visualisation. Brain wave sonification.

\section{INTRODUCTION}

Analema Group (2010) is an interactive art collective with a specific interest in telepresence, interactive and generative arts, scientific and artistic visualisation and the effect on audience members of human interaction. As an ongoing research subject of telepresence and its effect on human mind (Gingrich et al. 2013), the collective's new project looks at ways of directly investigating the effect of remote interaction onto the nervous system. The rich history of telepresence art creates the conceptual context for this piece. On an analytical level, we are interested in combining tools for quantitative measurements of interaction, with means to visualise and sonify this experience.

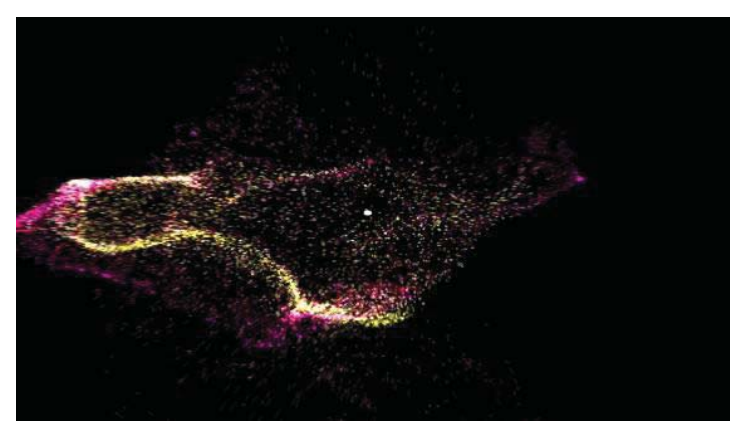

Figure 1: Visualisation test transmission

With "Transmission", the research collective implements an infrastructure to externalise brain activity. Transmission finds new strategies to visually represent this subjective experience and to create a sonic outlet for this activity. Users are invited to wear an emotive EEG headset, which allows them to see a visual representation of their own neural processes. A user in a remote location can control this visual representation and influence it. Through motion sensor (Kinect or Motion Analysis) and motion control techniques, a remote user hereby reacts to the sonification of someone else's brain activity. The remote user is allowed to modify its visual representation in real time, thus providing the wearer of the EEG headset with motion information. Such technology augmented telepresence, provides both users with an infrastructure to playfully explore a feedback loop of action and interaction. Brainwave activity can further be recorded and measured for research analysis. A team of experts in telepresence (Renaud, Zhidong Xiao), works with artists (Eugenia Emets), a producer (Gingrich), animators and programmers on the visual and sonic implementation of this conceptual framework.

\section{CONCEPT}

Similarly to Kima - the collective's most recent piece - the concept of Transmission is rooted in the history of telepresence art, performance art and generative art. Through the use of EEG data modification and its audio-visual interpretation, users are invited to visually and sonically explore their own brain activity in real time, thus developing 
a new language that engages their senses on multiple layers. This allows users to learn about the reactivity of their own nervous system. Transmission engages the user on conscious and subconscious levels and externalises the internal experience of presence in a remote location. Transmission redefines the boundaries of historical interactive kinetic art and its current developments through use of 3-dimensional holographic immersive projection technologies, neurobiological feedback and telepresence communication.

Interactive arts have been interested in shifting points of perception by engaging audiences in kinetic activities since Marcel Duchamp's "Rotary Glass Plates" (Duchamp 1920) and "Disks Bearing Spirals" (Duchamp 1923). Interactive arts increment chain reactions of cause and effect translating these relationships into kinetic action. Transmission is interested both in external body movement and internal activities of the user's brain. Transmission engenders a dialogue that is both conscious and subconscious at the same time. Kinetic movement is understood as a real-time interplay between one's mind and the other's body. Users never get to physically see each other; they explore their presence through remote interaction. Ultimately, we are investigating a sense of presence, of being there, being elsewhere - and what affect this presence has on another person, as well as on themselves and their own mind.

Since Roy Ascott, Paul Sermon and Tina Keane's pioneering experiments in telepresence art, the genre has experienced dramatic changes, a new focus on kinetic interactions and an integration of neighbouring disciplines through the development of technological aids such as easy motion tracking, programming languages for real-time visualisation and sonification of such cues (processing, Max/MSP, OSC, VVVV, etc.). Telepresence art has always been linked to media art, the frontiers of technology and interactive arts.

Transmission is the second project by Analema Group exploring sound and its effect on telepresence. Kima, our most recent piece, was concerned with the immersive aspect of sound and its effect on telepresence experiences, as well as its visual aspect and its role in communication. Transmission looks at sonic interpretation of telepresence effects on internal phenomena by externalising biological activity. Sound is the integral binding link, which not only embeds the visual representation, but also pre-empts and generates its form. Brain wave activity is digitally modified, so certain effects of entrainment can be explored. The user's mind will not simply listen to, but will actively create a sound-scape.

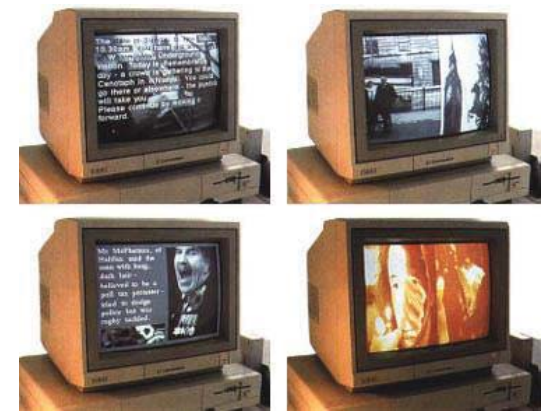

Figure 2: Paul Sermon: Think about the people now, 1998

Sound artists have explored resonance of telepresence on music for decades (for instance, Pauline Oliveros Deep Listening Institute). Concurrently, the relationship between music and neural phenomena was investigated since the 1960s (for example, Alvin Lucier's "Music for solo performer"). As EEG headsets became more affordable, its use within the arts increased. Sophisticated patches and interfaces for brain wave sonification have been developed for instance by Mick Grierson from the Department of Computing at Goldsmith University. Contemporary artistic discourse continues to include elements of brain wave interpretation, Lisa Park's "Eunoia" used brain activity to create cymatic experiment of water vibration.

Academic research on the intrinsic link between sound and brain activity is relatively scarce. Dr. Jeffrey Thompson studied brain reaction to specific frequencies. Transmission wants to facilitate active engagement with sound frequencies, the body as instrument, and music as a bi-directional, interactive experience. Transmission stands in a multitude of traditions, sound art, interactive art and most of all telepresence art. The research collective is interested in cross-overs between art and science as a two way stream: for the audience to experience new understandings on dynamics of our neural landscape, but also for science to potentially profit from new visualisation techniques.

This project finds inspiration in the therapeutic practice of neurofeedback applications. Evgenia Emets has conducted an exploratory study of sound frequencies effecting EEG streams during sound therapy sessions during her Diploma in Sound Therapy at the British Academy of Sound Therapy. Her previous work incorporated methods of neurofeedback into interactive art performances, i.e. A-Field (2010). Analema Group plans to use artistic visualisation and sonification to improve the quality of neurofeedback in therapeutical applications in collaboration with neuroscientists and neurofeedback practitioners. Lastly, this project is an academic project, a research project on telepresence. Alain Renaud and Oliver Gingrich 
investigate cueing parameters to measure, quantify and analyze the notion of presence.

\section{ACADEMIC RESEARCH}

With Transmission, we look at new, succinct model for the analysis of dimensions telepresence. Along with presenting a new concept for the analysis of telepresence, Analema Goup is exploring strategies of testing this model.

A multitude of classification systems exist for telepresence. For Lombard \& Ditton (1997), these include presence as social experience, presence as realism, presence as immersion, and presence as transportation among others. Sheridan identified five factors - three technology related factors and two context-based factors. Renaud (2010) distinguished between three types of cues for phonetic telepresence (behavioural, notational and temporal).

Sadowski \& Stanney (2002) distinguish between individual and system variables including ease of interaction, user initiated control, pictorial realism, and length of exposure, social factors and system factors. Freeman argues that the majority of the discourse can be narrowed down to up to three factors: immersion (Barfield \& Weghorst 1993, Prothero \& Hoffman 2007), involvement (Hoffmann et al. 1998) and realism (Barfield \& Hendrix 1995). Investigating presence as a complex phenomenon across only a few key dimensionalities allows for a degree of accountability and ultimately the potential to quantify presence through standardized tests.

As a multi-sensory and highly complex phenomenon, we are interested in a conceptualisation that allows for cross-platform applicability and a high degree of flexibility. Telepresence has changed dramatically from its original robotic conception. As telepresence became a function of everyday life, new models for cross platform analysis are needed. Our classification system is egocentric, subjective and puts the user and his or her individual perceptual experience at the heart of the analysis. Virtuality is conceptualized as a subcomponent of interactivity: for us in digital environments, 3D display technologies are a mediating presence as much as networked environments, mobile phones and other handheld devices. However, the form and technical modalities of these interactions are secondary. The concept of perceived non-mediation is central to our understanding of presence. More than dislocation or computer-human interaction presence is a multi-sensory interaction of perceived non-mediation within an immersive environment. We can measure both: subjective degrees of immersion and degrees of interactivity for the user and we can look at factors of realism. Consolidating various different positions and angles, there is a need for a simplified method of analysis. Different models of presence all share their concentration on involvement/ interactivity and immersion of the user and it is on this subjective level that we want to approach the phenomenon.

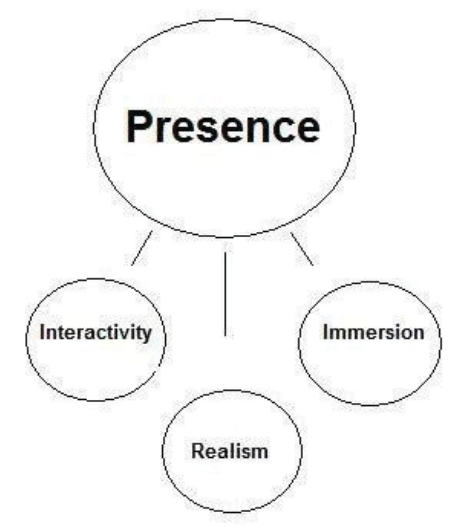

Figure 3: Standard telepresence model

Immersion is seen as both a perceptual and psychological phenomenon, as a physical reality and a subjective condition. Immersion encompasses a degree of absorption into a mediated environment, the exclusion of the outer world or the dominance of the media reality. Immersion can be both physically measured by looking at the amount of senses addressed through a media environment. Immersion is not a condition, but a co-factor of presence.

Interactivity is not only a technical condition, it is a perceptive reality, a potential of personal engagement and of the user's personal involvement. We are looking at interactivity as a cofactor for user's perception of remote presence, as a perceptive condition that is facilitated through technological cues. Lombard and Ditton (1997) conceptualised presence as social richness, a phenomenon underpinned by social presence theory by Short et al. (1976) and media richness theory (Rice 1992). The concept of interactivity is deeply related to questions of intimacy and immediacy. We will discuss cues for interactivity that ultimately contribute to the perception of presence.

With Transmission, we want to look at telepresence as both a subjective (immersion) and social (interactivity) experience. It is in the dialectic framework between individual experience and social activity that telepresence needs to be analysed. A combination of both qualitative data (subjective experience) and quantifiable data (EEG data) will help us to look at both components of telepresence - interactivity and immersion in all detail. As part of this research, we look at different 
audio and visual cues and their effect on both aspects of telepresence. The combination of a set of representative set of EEG data and correlating qualitative, standardised tests will help us to investigate the multi-dimensional field of immersion and interactivity, the complexities of the model itself and underlying cues and factors of statistic relevance. Transmission compares quantitative and qualitative research results, gathers feedback from audience and performers' participation through EEG recordings and questionnaires.

Visualisation projects on human brain activity and the nervous system such as The Human Connectome Project or The Human Brain Project look at visualisation and exploration strategies of the human brain. Transmission provides an artistic interpretation of brain wave information that can ultimately lead to introspective, therapeutic and possibly medical use.

\section{TECHNICAL SETUP}

Transmission uses two dedicated spaces:

- a motion capture studio for real-time transmission - the holographic Musion studio for display and to generate visual 3-dimensional interpretation of neurofeedback to control the motion stream and to generate a phonetic environment

Transmission's technical installation is described below. Motion data is controlling a particle stream displayed on holographic Musion interface in a remote location. We are generating both sound and visual data as well as motion data in real time, allowing for its modification, and thus a constant dynamic interplay between these three factors.

For testing purposes, the motion capture studio at Bournemouth University is linked up to the holographic studio at Musion where the audience can observe and interact with the representation of their own neural performance as a "holographic avatar".

Musion specialises on large scale Pepper's Ghost displays, which allows for "holographic" (a descriptive term) display through the illusion of 3D using optical cues. Holographic interfaces have the advantage of allowing for a certain cinematic, almost immersive experience, which in turn should facilitate our aim for enhanced social engagement with the technical environment. Brain wave data generated within this experience will be sonified in real time around the Musion stage area via surround sound technology. The resulting immersive character of the audio experience is in line with the visual qualities of the interactive display.
A visual and phonetic feedback signal is relayed from the Musion stage to the remote performer in the motion control studio at Bournemouth University. We have tested minimum motion control data speed with a threshold of $6-8 \mathrm{mbs}$ upload and download speed.

The Motion Analysis Cortex server at Bournemouth University is in charge of interpretation of the motion data in the motion control studio. Data from the Cortex server is passed onto Autodesk Maya via OSC (Open Sound Control) signals in real time. The particle stream will be generated based on this OSC signal input. On the holographic stage, an audience member will interact with this particle streams.

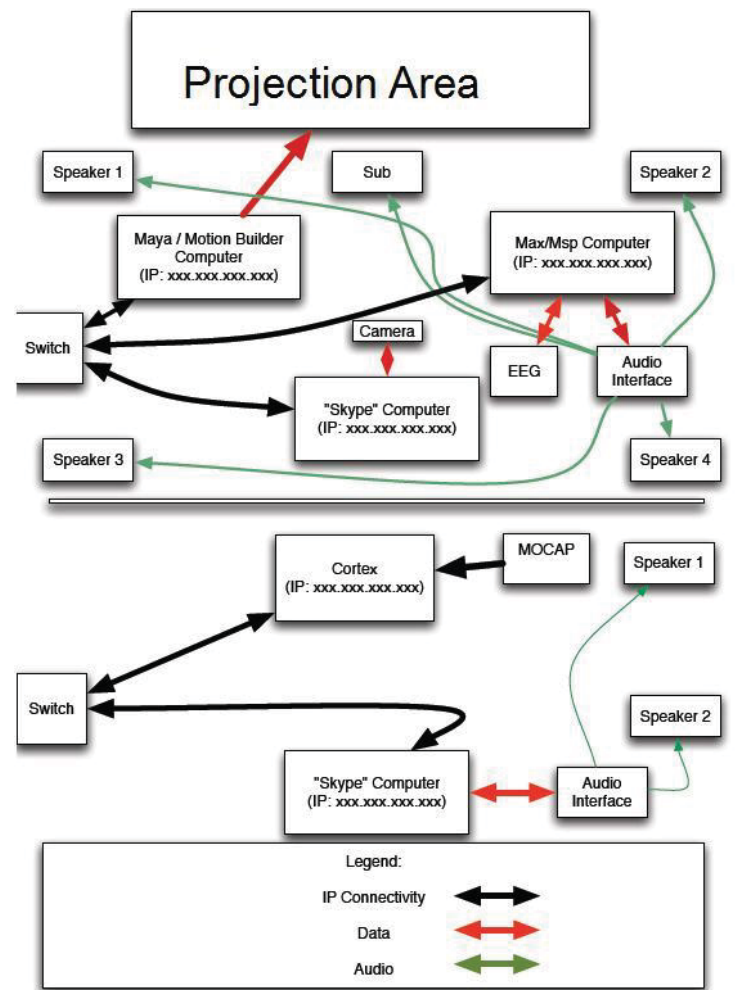

Figure 4: Model of the setup

An EEG device (Emotiv) analyses brainwaves of an audience member building a synapse between projector and transferred image. A stream of particles as complex animation visually represents the users' neural activity and provides them with valuable information on their inner processes. EEG data is also translated into sound. Several parameters such as different neural frequency bands and wavelengths can be mapped to visual representations and sound interpretations. Both visual and audio information are relayed via networks and as real time audiovisual signals to a user in a remote location, who reacts to this data. Generated imagery as well as the EEG data can be registered for later analysis. 
In Transmission, we attempt to use neuro-feedback methods to give participants tools for understanding their own neurological activity. A biofeedback loop not only consists in a visual mirror image of telepresence, on a sonic level, it enables the participant to control the sound scape in the room though relaxation and focused attention. Users control visual representation of the nervous system through colour codes, particle emissions and particle forces, they are also immersed in a sonic experience, an environment created by their own mind. Ultimately, we envisage including the visual field around the core "avatar" on the holographic display as an extension of the performance space. The sound will be generated as real-time sonification, as interpretation of distinctive brainwave patterns, i.e. a combination of dominant brainwave frequency bands. This procedural sound scape will be altered by gradual discrepancies in the EEG data values - similar to neuro-feedback signals (beep tones) in a medical or therapeutic environment. In Transmission, the users are hearing "sonic events", such as sound bursts, gentle vibrations - thus further elevating the mood and giving clear indication on the type of brainwave activity, i.e. relaxation, deeper states, concentration.

Conceptually, Transmission concentrates on the brain-body dichotomy - asking related questions: Is the mind located in the brain? How do we react to external presence - especially if this presence is only mediated, not visual and not representational? What effect does presence have on our neurological activity? Human neural activity can be affected by the presence of another person brainwave frequencies and heart rates fluctuate, when sitting in proximity or through holding hands with another person. Transmission visually and sonically explores the phenomenon of presence, allowing us to analyse its effects. Transmission provides participating users with an interface for playful engagement with their own brain.

In recent years dedicated EEG devices have been developed which provide both OSC signals as well as easy and flexible use for a mass audience. Neurosky, for instance, offers direct outputs to Max/Msp for parameter extraction. Emotiv sends OSC signals, in our case parsed onto a MEL script in the Autodesk Maya animation bundle. These open sound control messages then trigger complex particle effects as $3 d$ animation of the human nervous system visualizing human perception in real time.

A second user further adapts, changes and alters these signals on a visual level. This second user creates kinetic information that directly influences the EEG representation, The environments are equipped with relay signals to allow for real time references both in the motion capture studio and as holographic display in the environment of the EEG data emitter.

A number of tests have been conducted to ensure technical feasibility and to test limitations of the system.

Preliminary technical feasibility tests have been conducted between Musion Systems and the motion capture specialists 'The Imaginarium' at Ealing Studios London and Audiomotion in Oxford.

Initially, motion control system has been tested as input devices for the holographic screen display in combination with Musion's telepresence and Audiomotion Oxford in December 2012. Specific recording and display parameters were identified such as specific camera angles, optimum lighting in recording and display environments, frame rate and streaming latency. As a result, a certain degree of latency (of up to 5 frames per second) is anticipated.

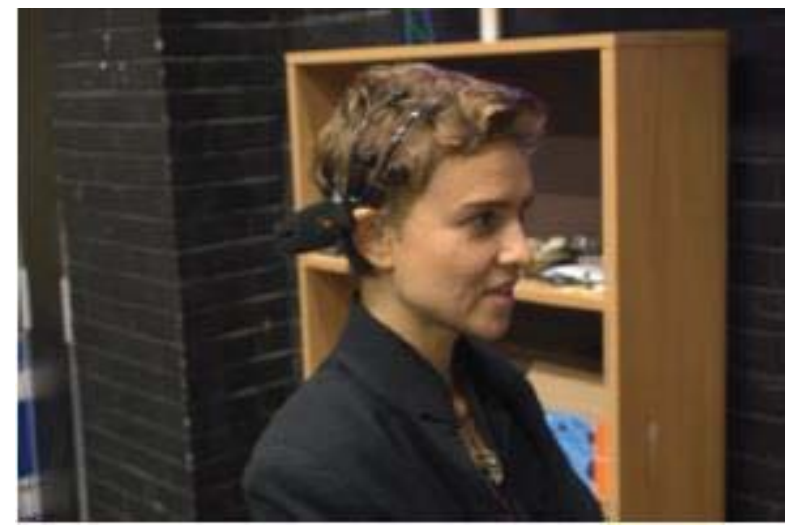

Figure 5: Motion capture test

Using a Vicon system and a Blade 2 engine for motion recording, we looked at complex motion data capturing of up to 60 cameras. In addition, both facial data and additional body information (fingers) were displayed in real time on the Pepper's ghost display screen for one or two performers. Limits such as overlap and occlusion between two or many performers were tested. The rig used 40-60 Vicon raptor cameras for maximum flexibility with multiple performers on the Blade 2 engine.

A second test, between The Imaginarium at Ealing Studios, and Musion on Portland Place was setup to test real time transmissions, latency, optimum workflow for data transmission as well as voiceover IP (VOIP) transmission. We identified a minimum transfer speed of $6-10 \mathrm{mbs}$ up and downlink. The motion capture studio at Bournemouth University is using Motion Analysis 
with a Cortex. We have tested transmission, OSC compatibility (script developed by Paolo Emilio Selva), and software integration. We will require a less complex technical setup than during the test rigs as only a single performer will be captured in quite a confined space, from a fixed viewing angle. The use of a Kinect camera device is conceivable Body occlusion is therefore impossible in this environment, resulting in the need for fewer cameras. Transmission does not make use of facial capture, fingers or any complex geometry.

After careful comparison between different EEG systems, we have opted for a flexible, cost-effective unit that has been successfully deployed in artistic contexts (Emotiv). Each and every performance requires a degree of improvisation and will largely depend on user interaction of the audience member participant. The user's brain wave will generate telepresence of the remote live performer - represented through his avatar on stage. Impulses drive the avatar's visibility, like currents of electric supply. Combining two data input sources of two distinctive spaces of both internal (neural) and external, kinetic motion in a single telepresence installation. The abstract visual representation of the human nervous system on a live stage provides a mirror image of and to the visitor - as a real time interface and visual display of the minds reaction to telepresence. The parameters of this interaction will not only be analysed through our research, but will also present the user with feedback on their own reactions.

\section{FUTURE AND OUTLOOK}

'Transmission' will be presented to a broad audience to museum spaces with a relevant interest in arts and science. In addition to realizing Transmission as performance piece with audience participation, we want to optimise Transmission as a permanent installation. As a performance piece a professional dancer will be invited to act and interact with users in real time. Sound modulation will be reacting live to the users' brainwave frequency data.

As a permanent installation, users are invited to explore telepresence between two spaces potentially using two headsets and two motion capture devices. A single user mode is also planned. On an artistic and conceptual level, Transmission tries to build on academic and medical research by focusing on the brain as a permeable, interactive and reactive source that operates in constant dialogue with the external world.
We do envisage secondary audience in the field of neurofeedback therapy, as we intend to test the resulting audio-visual interface with recipients of neurofeedback practice. We plan to introduce our visualisation and sonification methods into a therapeutic setting through direct engagement with experts in the field. We are directly involving therapeutic and medical experts in the development of the project and are intending potential medical uses as final applications for this project.

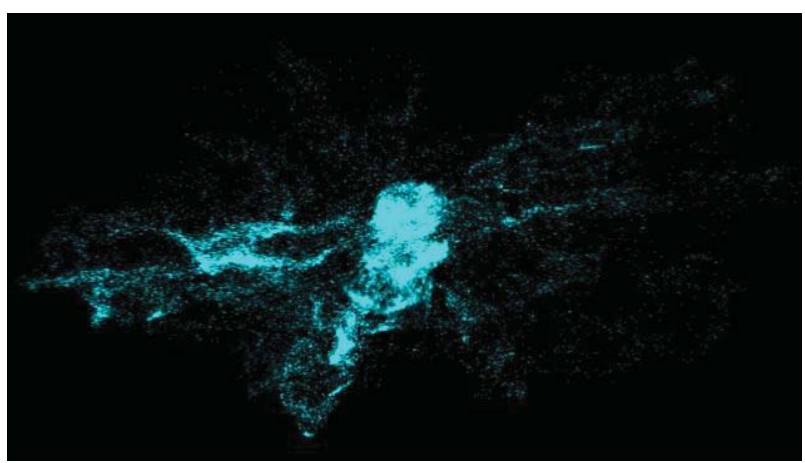

Figure 6: Visualisation test transmission

Academic research output of the project will aim for a more refined, and succinct model for telepresence analysis as well as a deeper understanding of underlying audio-visual cues and their effect on users. The generation of quantifiable EEG data, in conjunction with standardised telepresence questionnaires will result in a complex research outcome that will be further analysed and should contribute to a more comprehensive conceptualisation of telepresence.

On an artistic level, Transmission's declared aim is to offer a new tool for interactive engagement and a new, self-reflective experience of communication. Through creating an immersive space, we externalise an internal experience of dialogue, a sound scape of inner perception and an everchanging visual representation of stimuli of the other. Juxtaposing kinetic, physical movement with invisible, psychological impulses creates a feedback loop of exchange between the two, allowing audience to reflect on their perception of the internal vs external, the body and the mind. Transmission potentially leads the way to extended artistic exploration on perception and understanding of consciousness through new tools of interaction.

\section{REFERENCES}

Analema Group (2010) A-Field. Site specific Performance. London, UK.

Ascott, R. (1983) La Plissure du Texte Mixed Media. 
http://alien.mur.at/rax/ARTEX/PLISSURE/plissure.h $\mathrm{tml}$ accessed 24.March 2014.

Barfield, W. and Weghorst, S. (1993) The Sense of Presence Within Virtual Environments: A Conceptual Framework, in Human-Computer Interaction: Software and Hardware Interfaces, vol B, pp. 699-704

Barfield, W. and Hendrix, C. M. (1995) The effect of update rate on the sense of presence within virtual environments, Virtual Reality: The Journal of the Virtual Reality Society, vol. 1, no. 1, pp. 3-16.

Dove, T. (1994) Theater without actors: Immersion and response in installation. Leonardo, vol. 27, no. 4.

Duchamp, M. (1920) Rotary Glass Plates, Paris.

Duchamp, M. (1923) Disks Bearing Spirals, Paris.

Freeman, J. (2009) Implications for the measurement of presence from convergent evidence on the structure of presence. In C. C. Bracken and P. Skalski (eds.), Telepresence and Video Games: The Impact of Image Quality, PsychNology Journal, vol. 7, no. 1, pp. 101-112.

Gingrich, O., Emets, E., Renaud, A (2013) Enhancing presence - Immersive sound environments as presence generating factor. In $\mathrm{K}$. $\mathrm{Ng}$, J. P. Bowen, and S. McDaid (eds.), EVA London 2013: Electronc Visualisation and the Arts, pp. 19-23. British Computer Society, Electronic Workshops in Computing.

Grau, O. (2003) Virtual Art: From Illusion to Immersion. MIT Press, London.

Grierson, M. (2008) Composing with brainwaves: Minimal trial P300b recognition as an indication of subjective preference for the control of a musical instrument. Proceedings of the ICMC, Belfast.

Hayles, N. K. (1999) How We Became Posthuman. Chicago University Press. Chicago.

Hoffman, H.G, Prothero, J.D., Wells, M., Groen, J. (1998) Virtual Chess: The role of meaning in the sensation of presence. International Journal of Human-Computer Interaction, vol. 10, pp. 251-263

Keane, T. (1998-2003) Couch, Mixed Media. Monitors, Telepresence Environment \& Couch. Variable dimensions (Multiple Locations). http://www.luxonline.org.uk/artists/tina keane/couc h.html accessed 05. January 2014.

Liu, Y. and Shrum, L. J. (2002) What is interactivity and is it always such a good thing? Implications of definition, person, and situation for the influence of interactivity on advertising effectiveness. Journal of Advertising (4) M.E. Sharp. New York.

Lombard, D. and Ditton, T. (1997) At the heart of it all. The concept of presence. Temple University. Philadelphia.
Lucier, A. (1965) Music for a solo performer. Live Performance. Brandeis University.

Menache, A. (2000) Understanding Motion Capture for Computer Animation and Video Games. Morgan Kaufmann.

Minsky, M. (1980) Telepresence. Omno, pp. 45-51.

Oliveros, P. (2009) Deep Listening. (2009) http://deeplistening.org/site/content/pauline-oliveros accessed 21.December 2013.

Park, L. (2013) Euonia. Performance. http://www.thelisapark.com accessed on 22.March 2013.

Prothero, J.D. and Hoffman, H.G. (2007) Widening the field-of-view increases the sense of presence immersive virtual environments. In J. J. Lee (ed.), Human-Computer Interaction. $\mathrm{HCl}$ Intelligent Multimodal Interaction Environments, Springer, Lecture Notes in Computer Science, vol. 4552, pp. 393-400.

Renaud, A. (2010) Dynamic cues for network music interactions. In Proceedings of the SMC Conference.

http://smcnetwork.org/resources/smc2010 accessed 20.March 2014.

Rheingold, H. (1991) Virtual Reality: The revolutionary technology of computer-generated artificial worlds - And how it promises to transform society. Touchstone.

Rice, R. (1993) Media appropriateness: Using social presence theory to compare traditional and new organizational media. Human Communication Research, vol. 19, pp. 451-484.

Sadowski , W. and Stanney, K. (2002) Measuring and Managing Presence in Virtual Environments. University of Central Florida, USA.

http://web.cs.wpi.edu/ gogo/courses/imgd5100/pap ers/Sadowski HVE 2002.html accessed 20.March 2014.

Sermon, P. (1992) Telematic Dreaming, Mixed Media. Telepresence projection. Variable dimensions. Finland.

http://www.paulsermon.org accessed 15.February 2014.

Sheridan, T. (1992) Musings on telepresence and virtual presence, Presence, vol. 1, no. 1, pp 120126. Massachusetts

Short, J. A., Williams, E., and Christie, B. (1976) The Social Psychology of Telecommunications. Wiley. London

The Human Connectome (2014) http://www.humanconnectomeproject.org accessed 21.February 2014. 
The Human Brain Project (2014)

http://www.humanbrainproject.eu accessed on 15.February 2014.

Thewlis, D., Bishop, C., Daniell, N. and Paul, G. (2013) Next-generation low-cost motion capture systems can provide comparable spatial accuracy to high-end systems. Journal of Applied Biomechanics, vol. 29, no. 1, p. 112.

Thompson, Jeffrey (2013).

http://www.neuroacoustic.com accessed March 2014.
Xiao, Z. (2009) Motion Capture based Motion Analysis and Motion Synthesis for Human-like Character Animation. PhD thesis, Bournemouth University, UK.

http://eprints.bournemouth.ac.uk/14590/ accessed on 18 May 2014.

Zhang, Q. and Quo, X. (2013) 3D human motion retrieval based on human hierarchical index structure. Biolology of Sport, vol. 30, pp. 145-151. doi: $10.5604 / 20831862$ 